\title{
COMMENTARY
}

\section{Syndecan 4 Signaling and Intervertebral Disc Degeneration}

\author{
Fackson Mwale
}

From the Division of Orthopaedic Surgery, Lady Davis Institute for Medical Research, McGill University, Montreal, Quebec, Canada

Low back pain is a major problem in our societies, with a lifetime prevalence of $>80 \%$. One of the factors linked to low back pain is intervertebral disc degeneration (IVDD). Despite the significant impairment associated with IVDD, the mechanisms involved are poorly studied. The primary cause of IVDD is attributed to proteolysis by members of the matrix metalloproteinase (MMP) and a disintegrin and metalloproteinase with thrombospondin motifs (ADAMTS) families of enzymes. Enhanced expression of MMPs 1, 2, 3 , and 13 has been found in IVDD. Specifically, MMP-3/ stromelysin-1 has emerged as the key enzyme in the disc, where it is predominantly expressed and activates other MMPs, such as MMPs 1, 7, and 9. ${ }^{1}$ MMP-3 is upregulated in early osteoarthritis (OA) and in disc disease by cytokines.

In the current issue of The American Journal of Pathology, Wang et $\mathrm{al}^{2}$ describe the mechanisms by which cytokines, such as tumor necrosis factor- $\alpha$ (TNF- $\alpha$ ) and IL- $1 \beta$, control expression of MMP-3 in nucleus pulposus (NP) cells. The findings that the expression of MMP-3 is regulated by these cytokines and that syndecan 4 (SDC4), a cell surface proteoglycan, is vital to mediate this effect highlight the importance of understanding signaling pathways used by inflammatory cytokines in IVDD. These findings open new avenues to explore novel targets for therapies to moderate the catabolic effects of MMP-3.

\section{Disc Degeneration}

There are different mechanisms that contribute to IVDD, including reduction in nutrient supply, diminished cell viability, loss of notochordal cells, genetics, cell senescence, and loading history. ${ }^{3}$ Degeneration increases with IVD aging $^{4,5}$; the disc does not have endogenous repair activity. During disc degeneration, the NP changes from a gelatinous structure to a more fibrous texture as fissures appear in the NP and the annulus fibrosus (AF). With increasing age, there is a marked decrease in proteoglycan/collagen ratio [measured as glycosaminoglycan (GAG)/hydroxyproline ratio], which declines to approximately 5:1 in elderly persons (aged 60 to 80 years). The proteoglycan/collagen ratio has been reported to be highest in patients with grade 2 discs (approximately 23:1) and lowest in patients with grade 4 discs (approximately 5:1). ${ }^{5}$ In healthy juveniles (aged 2 to 5 years), the GAG/hydroxyproline ratio in the NP is approximately 25:1. Similarly, in young adult disc with no degeneration (aged 15 to 25 years), the GAG/hydroxyproline ratio within the NP is approximately 27:1. The extracellular matrix (ECM) undergoes biochemical changes in composition and structure because of the reduced synthesis and enhanced degradation. Proteolytic damage mostly affects aggrecan. Wang et $\mathrm{al}^{2}$ suggest that it may be possible to slow the progression of IVDD and proteolysis.

MMPs are involved in ECM turnover and disc degeneration. ${ }^{6}$ MMPs are broadly divided into the following groups on the basis of substrate specificity and cellular location: collagenases (MMPs 1, 8, and 13), stromelysins (MMPs 3, 10, and 11), gelatinases (MMPs 2 and 9), membrane-type MMP, and adamalysin (ADAM and the subgroup ADAMTS). Two members of this family (ADAMTS 4 and 5) degrade aggrecan and have been termed aggrecanases.

Disc degeneration is characterized by an imbalance in the production and activation of MMPs relative to their inhibition by tissue inhibitors of metalloproteinases (TIMPs).

\footnotetext{
Supported by AO Spine, Canadian Institute of Health Sciences, and North American Spine Society.

Disclosures: None declared.

Address correspondence to Fackson Mwale, Ph.D., Division of Orthopaedic Surgery, Lady Davis Institute for Medical Research, McGill University, 3755 Chemin de la Cote Ste-Catherine, Montreal, QC, Canada H3T 1E2. E-mail: fmwale@1di.jgh.mcgill.ca
} 
This leads to an inflammatory milieu, which favors the synthesis of proinflammatory cytokines. In addition to cytokines, other inflammatory mediators, such as nitric oxide and eicosanoids (prostaglandins and leukotrienes), also play a major role in IVDD. In contrast, transforming growth factor- $\beta$ (TGF- $\beta$ ) is known to increase proteoglycan synthesis and counteract cytokine-mediated suppression of matrix synthesis.

Substrates of stromelysins include proteoglycans, fibronectin, elastin, laminin, and several collagens. By using a genetic approach, Wang et $\mathrm{al}^{2}$ report the mechanisms by which TNF- $\alpha$ and IL-1 $\beta$ control expression of MMP-3 in NP cells. MMPs 3, 7, and 12 are the most efficient MMPs at cleaving aggrecan, whereas ADAMTS5 is more efficient than ADAMTS4 at cleaving within the aggrecan interglobular domain. Expression of MMP-3 can be enhanced by increasing strains and is reduced by IL-1 receptor antagonist protein in degenerated and herniated human tissues. Similarly, monoclonal antibodies against TNF- $\alpha$, the p38 mitogen-activated protein kinase inhibitor, suppress the expression of MMP-3 and IL-1 in explants of herniated discs, ${ }^{7}$ whereas an NF- $\mathrm{KB}$ decoy decreases pain in a rat lumbar disc herniation model. ${ }^{8}$

\section{IVD, Proteoglycans, and Collagens}

IVDs are formed of the peripheral fibrous ring (AF) surrounding the proteoglycan-rich central NP, sandwiched between cartilaginous end plates. ${ }^{9}$ The function of the disc is to resist compression and permit limited movements in which loading forces are spread evenly on the vertebral bodies. The cell density in the adult NP is $4 \times 10^{6}$ cells $/ \mathrm{cm}^{3}$, whereas the AF has $9 \times 10^{6}$ cells $/ \mathrm{cm}^{3}$, which is low compared with hyaline cartilage, suggesting that few cells maintain homeostasis in a vast ECM. The IVD serves to support mechanical loads via hydraulic and osmotic pressures. These properties are because of the high concentration of proteoglycans (predominantly aggrecan) entangled in a dense network of collagen fibers and noncollagenous proteins in the ECM. ${ }^{10}$

\section{Growth Factors with the Potential to Dampen the Up-Regulation of MMP-3}

Maintaining disc homeostasis requires a careful balancing act through complex anabolic [bone morphogenetic proteins (BMPs), TGF- $\beta$, and insulin-like growth factor] and catabolic (MMPs, aggrecanases, and inflammatory cytokines) factors, in addition to TIMPs, which control catabolic activities. ${ }^{11}$ Therefore, inhibition of proteolysis has emerged as a tempting therapeutic approach in IVD repair. Other therapeutic molecules, apart from the anabolic ones, such as anti-catabolics (TIMP1 and TIMP2) and intracellular regulators (Smad proteins and Sox 9), are being used. ${ }^{12}$

TGF- $\beta$ regulates cell proliferation and ECM synthesis. TGF- $\beta$ is stored in a latent form, and little TGF- $\beta$ exists in the active form. Recombinant BMP2 can also stimulate proteoglycan synthesis in IVD cells. ${ }^{13}$ Others have found that LIM mineralization protein-1 can stimulate proteoglycan synthesis through its action on BMP. The BMP pathway consists of BMP dimers binding to a membrane complex composed of BMP receptors 1 and 2 (serine/ threonine kinases). Wang et $\mathrm{al}^{2}$ suggest that TGF- $\beta$ can block TNF- $\alpha$-induced up-regulation of MMP-3 by counteracting the NF- $\kappa \mathrm{B}$ pathway. It is possible that other anabolic growth factors outlined above could perform the same function.

\section{SDCs as Therapeutic Targets in Disc Degeneration and OA}

SDCs are transmembrane domain proteoglycans that can act as coreceptors. They possess a core protein with heparin sulfate and chondroitin sulfate chains, which permits the interaction with other cells, the ECM, and growth factors, such as TGF- $\beta{ }^{14}$ The perceived function of SDCs is to modulate the activation of receptors at the cell surface. There are four members in this family (namely, SDCs 1 to 4). Few studies have been conducted on SDCs in the disc and, thus, their function is not known. Central to all of the activities of SDCs are the binding of ligands to their receptors and the shedding of extracellular domains from the cell membrane in response to pathological conditions, which can wield stimulatory or inhibitory properties on signaling pathways. Although SDCs shed their ectodomains, few studies have addressed their significance. It is clear that shedding is by design and suggests that a role for the soluble proteoglycan ectodomains is required but must also be regulated. Wang et $\mathrm{al}^{15}$ showed an increase of SDC4 mRNA expression in disc degeneration and concluded that, in the NP, TNF- $\alpha$ and IL- $1 \beta$ regulate SDC4 expression, which promotes aggrecan degradation by ADAMTS5. Similarly, SDC4 is unregulated in human OA and regulates ADAMTS5 activation and cartilage degradation. ${ }^{16}$ The loss of SDC4 in genetically modified mice and intra-articular injections of antibodies to SDC4 into wild-type mice suppress cartilage damage in a surgically induced model of OA, suggesting that inhibition of SDC4 is a potential therapeutic strategy for the treatment of cartilage and disc degeneration. ${ }^{16}$ Despite these observations, the mechanisms by which cytokine-mediated MMP-3 expression accelerates the initiation and progression of IVDD are poorly understood. Earlier studies have suggested a mechanistic paradigm that links SDC4 and MMP-3 expression and activation.

\section{Summary and General Considerations}

IVD matrix turnover is mediated by a host of factors that could lead to remodeling, catabolic processes, or repair. Repair and maintenance of the IVD have been researched extensively, and considerable progress has been made. Alteration of these processes leads to abnormal signaling 
pathways that contribute to disc degradation. We now know that disc degeneration is a result of an abnormal response of cells to gradual structural failure. ${ }^{3}$ Indicators of altered cell metabolism are enhanced cytokine and MMP activities. ${ }^{6,17}$ The involvement and the role of inflammation are now widely accepted. In addition, emerging evidence suggests a link between MMPs, ADAMTS, and SDC4. ${ }^{15,16,18}$

In conclusion, Wang et $\mathrm{al}^{2}$ concentrate on signaling pathways controlling cytokine-mediated MMP-3 expression during IVDD. In establishing that the expression of MMP-3, which is regulated by TNF- $\alpha$ and IL- $1 \beta$ in NP cells, requires SDC4 and signaling through the p38 mitogen-activated protein kinase and NF- $\kappa \mathrm{B}$, the current study raises the possibility of a separate signaling event downstream of SDC4. Indeed, studies from this group had given some clues that suggested that SDC4 might be required for the expression and activation of MMP-3 by cytokines. Moreover, SDC4 expression is also influenced by TNF- $\alpha$ and IL- $1 \beta$ through NF- $\kappa B$, and it is postulated to stimulate ADAMTS5 activation, which might lead to aggrecan degradation. TGF- $\beta$ has been suggested to dampen the cellular response to TNF- $\alpha$ because it was found to block the cytokine-induced production of MMP-3. These advances will help in the elucidation of the target factors that are intimately involved in IVDD.

\section{References}

1. Liu J, Roughley PJ, Mort JS: Identification of human intervertebral disc stromelysin and its involvement in matrix degradation. J Orthop Res 1991, 9:568-575

2. Wang X, Wang H, Yang H, Li J, Cai Q, Shapiro IM, Risbud MV: Tumor necrosis factor- $\alpha-$ and interleukin- $1 \beta$-dependent matrix metalloproteinase-3 expression in nucleus pulposus cells requires cooperative signaling via syndecan 4 and mitogen-activated protein kinase-nuclear factor $\kappa \mathrm{B}$ axis: implications in inflammatory disc disease. Am J Pathol 2014, 184:2560-2572

3. Adams MA, Roughley PJ: What is intervertebral disc degeneration, and what causes it? Spine (Phila Pa 1976) 2006, 31:2151-2161

4. Antoniou J, Steffen T, Nelson F, Winterbottom N, Hollander AP, Poole RA, Aebi M, Alini M: The human lumbar intervertebral disc: evidence for changes in the biosynthesis and denaturation of the extracellular matrix with growth, maturation, ageing, and degeneration. J Clin Invest 1996, 98:996-1003
5. Mwale F, Roughley P, Antoniou J: Distinction between the extracellular matrix of the nucleus pulposus and hyaline cartilage: a requisite for tissue engineering of intervertebral disc. Eur Cell Mater 2004, 8: 58-63. discussion 63-4

6. Le Maitre CL, Freemont AJ, Hoyland JA: Localization of degradative enzymes and their inhibitors in the degenerate human intervertebral disc. J Pathol 2004, 204:47-54

7. Genevay S, Finckh A, Mezin F, Tessitore E, Guerne PA: Influence of cytokine inhibitors on concentration and activity of MMP-1 and MMP3 in disc herniation. Arthritis Res Ther 2009, 11:R169

8. Suzuki M, Inoue G, Gemba T, Watanabe T, Ito T, Koshi T, Yamauchi K, Yamashita M, Orita S, Eguchi Y, Ochiai N, Kishida S, Takaso M, Aoki Y, Takahashi K, Ohtori S: Nuclear factor-kappa B decoy suppresses nerve injury and improves mechanical allodynia and thermal hyperalgesia in a rat lumbar disc herniation model. Eur Spine J 2009, 18:1001-1007

9. Mwale F, Iatridis JC, Antoniou J: Quantitative MRI as a diagnostic tool of intervertebral disc matrix composition and integrity. Eur Spine J 2008, 17(Suppl 4):432-440

10. Mwale F: Collagen and other proteins of the nucleus pulposus, annulus fibrosus, and cartilage endplates. Edited by IM Shapiro, MV Risbud. The Intervertebral Discs. Springer, Vienna, Austria. 2013, pp 79-92

11. Mwale F: Molecular therapy for disc degeneration and pain. Global Spine J 2013, 3:185-192

12. Yoon ST, Patel NM: Molecular therapy of the intervertebral disc. Eur Spine J 2006, 15(Suppl 3):S379-S388

13. Kim DJ, Moon SH, Kim H, Kwon UH, Park MS, Han KJ, Hahn SB, Lee HM: Bone morphogenetic protein-2 facilitates expression of chondrogenic, not osteogenic, phenotype of human intervertebral disc cells. Spine (Phila Pa 1976) 2003, 28:2679-2684

14. Pap T, Bertrand J: Syndecans in cartilage breakdown and synovial inflammation. Nat Rev Rheumatol 2013, 9:43-55

15. Wang J, Markova D, Anderson DG, Zheng Z, Shapiro IM, Risbud MV: TNF-alpha and IL-1beta promote a disintegrin-like and metalloprotease with thrombospondin type I motif-5-mediated aggrecan degradation through syndecan-4 in intervertebral disc. J Biol Chem 2011, 286:39738-39749

16. Echtermeyer F, Bertrand J, Dreier R, Meinecke I, Neugebauer K, Fuerst M, Lee YJ, Song YW, Herzog C, Theilmeier G, Pap T: Syndecan-4 regulates ADAMTS-5 activation and cartilage breakdown in osteoarthritis. Nat Med 2009, 15:1072-1076

17. Le Maitre CL, Freemont AJ, Hoyland JA: The role of interleukin-1 in the pathogenesis of human intervertebral disc degeneration. Arthritis Res Ther 2005, 7:R732-R745

18. Fujita N, Gogate SS, Chiba K, Toyama Y, Shapiro IM, Risbud MV: Prolyl hydroxylase 3 (PHD3) modulates catabolic effects of tumor necrosis factor-alpha (TNF-alpha) on cells of the nucleus pulposus through co-activation of nuclear factor kappaB (NF-kappaB)/p65 signaling. J Biol Chem 2012, 287:39942-39953 